\title{
Training to improve patient-centered electronic health record (EHR) use
}

\author{
Cynthia J. Sieck ${ }^{1,2}$, Brian Henriksen ${ }^{3}$, Sara Scott ${ }^{1}$, Natasha Kurien ${ }^{1,2}$, Mark Rastetter ${ }^{1}$ \\ ${ }^{1}$ Department of Family and Community Medicine, College of Medicine, The Ohio State University, Columbus, OH, USA; ${ }^{2}$ CATALYST, Center for \\ the Advancement of Team Science, Analytics, and Systems Thinking, College of Medicine, The Ohio State University, Columbus, OH, USA; ${ }^{3}$ Fort \\ Wayne Medical Education Program, Fort Wayne, IN, USA \\ Contributions: (I) Conception and design: CJ Sieck, B Henriksen, M Rastetter; (II) Administrative support: None; (III) Provision of study materials or \\ patients: CJ Sieck, B Henriksen, M Rastetter; (IV) Collection and assembly of data: CJ Sieck, B Henriksen, M Rastetter, N Kurien; (V) Data analysis and \\ interpretation: CJ Sieck, B Henriksen, M Rastetter, N Kurien; (VI) Manuscript writing: All authors; (VII) Final approval of manuscript: All authors. \\ Correspondence to: Cynthia J. Sieck, PhD, MPH. Associate Professor, Department of Family and Community Medicine; CATALYST, The Center for \\ the Advancement of Team Science, Analytics, and Systems Thinking, The Ohio State University College of Medicine, 460 Medical Center Drive, \\ Room 524, Columbus, OH, USA. Email: Cynthia.sieck@osumc.edu.
}

\begin{abstract}
Background: Electronic health records (EHRs) are used across healthcare systems to reduce clinical care errors, improve care team communication, and enhance care coordination and patient safety. However, one criticism is that EHRs increase the provider's engagement with the computer and decrease engagement with the patient leading to less patient-centered care. Patient-centered care is personalized care tailored to individual patient needs and preferences. Interventions have been suggested to help manage EHR use during visits while balancing computer interaction and patient-centered care.
\end{abstract}

Methods: Using the Resident-as-Teacher: a layered learning intervention, patient care training, patientcentric EHR use and team development of residents is balanced with creating a shared understanding of these processes. New interns serve as scribes for the senior residents, observing how patient care is conducted while taking notes necessary for charting and billing requirements. The intern and senior resident together navigate the EHR to ensure proper documentation. In addition, attending physicians precept every patient, providing the aspect of layered learning. The roles are then reversed and the senior resident becomes the intern's scribe. The intern is able to focus on patient care without any distractions that would have been present if the intern was fully in charge of the visit. The resident addresses any missed items with the intern before conclusion of each office visit. EHR training in this manner resulted in $15 \%$ more patient encounters while building rapport between the residents. Our assessment included an examination of patient visit counts and an open-ended survey administered to all interns and residents. Adapting training to telehealth during COVID-19 highlights adaptations to in-person training that could be implemented in the virtual environment while maintaining connection between the preceptor and resident. For example, use of a "virtual precepting room", providing the most up to date best practice information and training residents how to provide the best possible care with the limited information received when only seeing the patient virtually. We conducted qualitative interviews with residents approximately one month into the training to assess residents' perceptions of its impact and support they received.

Results: Analysis of the Resident-as-Teacher suggests that it provided more patient interactions for interns and residents, as well as facilitated rapport building on the team. For adapting training to telehealth, interviews with residents noted a few challenges but support from attendings was appreciated.

Conclusions: EHRs have been viewed more as a system required in health care and less of a tool to aid in organization and communication. With appropriate training, EHRs can be an asset to clinical care while working in conjunction with patient-centered care. Providers working together during a resident training period can promote a beneficial learning curve for both patient care and reducing providers' amount of work after clinic. 
Keywords: Electronic health record (EHR); primary care; systems

Received: 27 August 2020; Accepted: 03 August 2021; Published: 25 March 2022.

doi: 10.21037/jhmhp-20-121

View this article at: https://dx.doi.org/10.21037/jhmhp-20-121

\section{Introduction}

Electronic health records (EHRs) were introduced with the goal of reducing errors in clinical care, and have been significantly integrated across all areas of health care. The benefits of EHRs include improved communication among care team members, enhanced care coordination and increased patient safety (1-3). However, the use of EHRs has been criticized as increasing the provider's interaction with the computer and decreasing the provider's interaction with the patient, thereby potentially leading to care that is less patient-centered. More specifically, Alami et al. identified significant and interconnected ways in which EHRs can contribute to challenges for care teams including a sense of depersonalization in clinical relationships, prioritization of administrative needs over patient-centered needs, additional stress and frustration for healthcare providers, and cognitive overload (4).

Patient-centered care emphasizes care that is personalized to the patient (5) and recognizes that this type of connection between a patient and physician is associated with higher physician satisfaction to an even greater extent than the physician's income or control over the environment results (6). Clinician interaction with the computer during a visit decreases eye contact and conversation with the patient, leading to less patient-centric care (7) and decreased patient engagement (8). Research suggests that patients participate less when their physician is focused on the computer. Best practices related to patient-centered EHR use, such as through screen sharing $(9,10)$, can facilitate engagement but examination rooms may not be arranged in ways that encourage screen sharing with patients. Even when this is possible, many physicians are unlikely to use this technique to engage patients and when they do, patients are less familiar with the EHR and may not understand the interface they see, thus limiting their ability to engage (4).

Despite these challenges, EHRs are now a part of the clinical care ecosystem and studies have identified actions that physicians can take to improve their interaction with both the patient and computer to provide more patientcentered care (11). For example, Fogarty et al. identified a range of communication enhancing behaviors including the use of mobile monitors that can be shared with the patient, achieving a sufficient level of typing that does not require looking at the keyboard, explaining to the patient what you are entering into the EHR, pointing to specific elements on the screen to encourage patient engagement and reserving routine data entry for non-visit time (12). This last suggestion, while allowing more focus directly with the patient during the visit, is challenging, particularly in primary care where the burden of documentation is high (13). For example, one study found that nearly half of the time primary care physicians spent using the EHR was geared toward non-patient-centered tasks such as documentation and chart review (14). Balancing these tasks with direct patient care, as well as other administrative clinical responsibilities (i.e., EHR inbox management) is a significant source of stress for providers (15).

While the challenges of operating in a patient-centric manner are present across all levels of physician experience, medical residents must simultaneously learn both to interact with patients and with the EHR. Studies suggest that students and residents vary in their level of comfort using EHRs and are more proficient in some areas than in others. For example, Lander et al. suggested that confidence was greater for retrieval of information than for documentation (16). In addition, while residents may understand the benefits that come from using an EHR, they find the experience frustrating (17) and may be unaware of how little they collaborate with patients during the exam (18).

Training residents to use the EHR in a patient-centric manner can influence their use of EHRs throughout their careers (17). However, a systematic review of training programs noted significant deficits. Rajaram et al. noted that training interventions ranged significantly in time devoted to EHR use from a single 60-minute session to a 5 -week simulated training program (19). In addition, while all programs included in the review offered hands-on use of the EHR, only 2 included the opportunity to use the EHR while conducting an exam with a simulated patient $(20,21)$. Further, most programs focused on documentation of the encounter, which, while an important element of 
a physician's training, does not directly address engaging with the patient while using the EHR. Focusing on the documentation and not patient engagement deters away from patient-centered care.

These factors described above contribute to the challenges of incorporating EHR use while respecting the need for good clinician-patient relationships. Healthcare systems and teaching institutions are taking important steps to address these issues. Below we highlight programs which focus on overcoming these challenges to facilitate both efficient EHR use and establish strong clinician-patient relationships.

\section{Methods}

The study was conducted in accordance with the Declaration of Helsinki (as revised in 2013). The interview component of the telehealth study was the only portion considered research and was approved by institutional/ regional/national ethics/committee/ethics board of The Ohio State University and informed consent was taken from all participants.

\section{Resident-as-Teacher: a layered learning intervention}

Early in residency training, when interns learn about both patient care and proper EHR documentation, there is an important opportunity to model EHR use that facilitates a focus on the patient. Understandably, it is a challenging time in training, as residents are expected to learn how to manage patients while also navigating new relationships in the hospital and program, and finding work-life balance. To novice users, the EHR is not intuitive, particularly for obtaining appropriate information on sensitive psychosocial issues. This interface presents further complications for the PGY1 residents who have not established a rapport with our midlevel providers yet. The EHR training we describe assists the new resident in setting up co-scheduled appointments with in-house support providers.

Layered on top of this, residents are often learning a new EHR system, charting requirements, billing code fields, and how to respond to numerous different messages. This busy time leaves little room for team building and establishing an esprit décor programmatically, yet these elements are critical to developing a supportive learning environment in which the EHR is viewed as a resource, not a burden.

Our project sought to balance resident training in patient care, patient-centric EHR use and the development of the residents as a team with a shared understanding of these processes. We implemented this project in a patientcentered medical home with an in-house pharmacist, social workers, and behavioral and mental health specialists. In this project, the new interns served as scribes for the senior residents for 2 weeks in our family medicine outpatient clinic. Intern scribes could see their seniors providing direct primary care, observe how the patient interactions are conducted, and take notes on each aspect of the encounter that would be needed for charting and billing requirements, learning both patient care and documentation but in a lower pressure environment. The intern-senior pair would then jointly navigate the EHR after the visit to ensure that everything was documented in the proper place. These interactions taught the interns about patient care and efficient documentation practices, which then reduced the amount of work after clinic for both the intern and the senior resident as it was a shared task. In addition, attending physicians precepted each and every patient, which provided the layered learning aspect of the project while also serving as an additional role model for the resident to observe.

After those initial 2 weeks, the roles were reversed and the senior resident became the intern's scribe. This role reversal allowed the intern to focus on the patient care and delivery aspect of each encounter without the distraction or interruptions that had potentially occurred when interns were fully responsible for the entire visit. Any items the intern may have missed were commonly caught by the senior resident and addressed with the intern before concluding the office visit with each patient. The post visit charting again utilized both residents and reduced the afterclinic work burden.

\section{Statistical analysis}

For the Resident-as-Teacher intervention, we conducted an independent $t$-test to assess the statistical significance of the difference in visits.

\section{Adapting training to telebealth during COVID-19}

The COVID-19 pandemic created additional challenges to engaging with both the patient and EHR as visits rapidly shifted to a primarily telehealth format. In these situations, physicians had to connect with the patient using video while still engaging with the EHR on their computer. During this transition, all physicians had to quickly learn elements of practicing via telehealth, and still maintain a patient- 
centered approach.

While rapidly learning to use telehealth technology to interact with patients and maintain engagement, faculty were also learning how to train residents using virtual media. Some elements were primarily technical such as arranging the viewing space to see both the patient and EHR. When using one monitor, the video dialogue screen can obscure portions of the EHR, making it challenging to document efficiently. Ensuring access to two screens to make telehealth viewing of these various components manageable is one example of technical improvements.

Other considerations focused more on adaptations to inperson training of residents that could be implemented in the virtual environment and still maintain the connection between the faculty and resident. One important action was to create a "virtual precepting room", created in a Health Insurance Portability and Accountability Act (HIPAA) secure manner and compatible with many platforms including Zoom (with appropriate platform and settings) and Microsoft teams. Residents were able to speak to their patients via a telehealth app and then check in to the virtual precepting room to discuss the patient encounter with their preceptor. The preceptor had the ability to see the patient's EHR and, if a preceptor needed to "see" or talk to the patient, they could then call the patient to verify information. This allowed the resident to focus on connection with the patient while receiving support from the preceptor as would happen in an in-person setting.

In the virtual environment, the training is not only focused on clinical decision making, but how to make decisions using the more limited information available via a virtual platform. This effort required coaching residents through how to obtain the correct history and what can be done without a traditional physical exam. Some solutions included having patients take pictures, for example of their rash, and upload them to their patient portal if the patient had limited video technology, or teaching the patient to utilize different musculoskeletal maneuvers to make the correct diagnosis.

We also realized that successfully launching a virtual platform required significant support from leadership to incorporate appropriate documentation and billing in the EHR. As many academic medical centers across the country were facing similar challenges, we looked to professional organizations such as the Society of Teachers of Family Medicine for guidance in this rapidly changing environment (22). In addition, drawing upon the experience of our Center for Primary Care Innovation and
Transformation (PCIT), we established rapid communication mechanisms to ensure faculty and residents received the most up to date best practices, including pushing notifications through the EHR, offering smartphrases and appropriate billing, as well as communication tips and guidance (23). For residents who are new to billing, our training focused on applying appropriate office-based codes to the virtual environment in which a physical exam is not possible. Use of the virtual precepting room was critical here to allow the resident to fully engage with the patient during the telehealth visit while still remaining in close consultation with the precepting physician.

Finally, the rapid switch to telehealth enabled us maximize the patient-centeredness of follow-up plans by incorporating a range of telehealth interventions. In this capacity, we focused resident training on expanding the view of follow-up to include more frequent but brief telephone or video check-ins rather than requiring in-person visits. Doing so required a focus on EHR documentation of the patient's constraints and preferences for telehealth or inperson visits. However, this type of documentation is not usually collected and highlights a deficiency in many EHR systems. Social determinants of health screenings do not include items assessing access to the internet or devices that support telehealth applications.

\section{Qualitative analysis}

We conducted 5 focus groups with up to 3-4 residents in each, for a total of 19 residents, to understand their perceptions related to training in this environment. Focus groups utilized a semi-structured focus group guide to structure the discussion.

\section{Results}

\section{Resident-as-Teacher: a layered learning intervention}

Compared to the number of patient encounters by interns in the previous year, those in the inter-senior pair had $15 \%$ more patient encounters. Although our $t$-test of this difference was not statistically significant $(P=0.36)$, these encounters nonetheless provided additional opportunities for residents to interact with patients. An open-ended participant survey revealed that by using the EHR as the mechanism for the intern-senior pairs to work from for joint patient encounters, the residents got to know each other better and built rapport more quickly. The peer teaching component fostered open communication regarding clinical care decision 
making before the plan was brought to attending physician for final review. This step was embraced by the residents because it allowed them to hone their decision-making skills with their supportive senior residents. This mentoring further helped the intern in building positive relationships with patients, nurses, attending physicians, and billing staff through improved documentation. The EHR served as a tool around which the reflections and relationships were built.

\section{Adapting training to telebealth during COVID-19}

To assess the impact of our virtual training efforts, approximately one month after our shift to telehealth, we conducted focus groups with our residents to understand their experiences in this environment. While few had experience with telehealth prior to COVID-19, and first year residents had little patient care experience, much of this setting was entirely new to our residents. Residents acknowledged the challenges of learning to focus on the patient, provide appropriate care and document accurately. For example, one resident told us, "I think in the beginning it was challenging because a lot of our rotations were off, we weren't seeing as many patients in person ... more so for the second and third years which led to some of our skills that we had like in-person skills on examining and admissions perbaps go down, but as, and also kind of our educational activities tend to decrease, but as we've progressed we've started to ramp things back up or we've innovated ways to do virtual education and we've had talks on like how to do exams over virtual health which has been helpful".

Despite these challenges, residents understood the necessity of the rapid change and felt supported in the process. As one resident stated, “... it was a lot of changes all at once but I think having good support from the attendings was definitely belpful throughout the process." Another described the support from attendings in this way, "... so we're like constantly pretty much connected with Zoom, so if anything comes up you can just like either pop on if you like went off for some reason or you can just reach out and they're available the entire time they're assigned there."

Ultimately, they viewed the experience as learning skills that would be useful throughout their careers. One resident summarized the experience as, "So I mean before this all started, absolutely unprepared in like what the idea consisted of. So I think the one nice thing is that we've gained kind of a lot of experience from this and if in the future we ever have it as an opportunity as part of our practice, I'll feel much comfortable doing it overall."

\section{Discussion}

The EHR has been viewed as a tool clinicians are required to use rather than an initiative driven into practice by clinicians (24). Healthcare personnel want to minimize mistakes and provide an avenue for the best possible patient outcomes, however use of the EHR presents challenges in maintaining connection to patients. Training on best practices for using this tool to facilitate patient interactions and care is a critical element of resident education.

When viewed from the perspective of a training tool, the EHR has the potential to become an asset to new physicians. If residents are taught how to use an EHR to their advantage and how to incorporate it into their practices in a patient centered approach, both for in-person and virtual clinical encounters, they will likely feel more comfortable and empowered. As with any tool, there is a time commitment to learning how to use it, and one EHR system is often not like another. Providers working together during a training period will promote a beneficial learning curve for both patient care and reducing the providers amount of work after clinic.

We argue that a perspective change and positive examples of an HER's application to promoting patient-centered care and relationships can be achieved for the tool, and its use, to reach its full potential and provide patient-centered care. The purposeful utilization of the EHR to build providerto-provider communication and expanding patient access to care are robust positive examples of what it could provide. However, it is important to consider that EHRs vary with respect to their ease of use and patient-centeredness. Resident training that seeks to increase patient-centered use of the EHR is still limited by the EHR itself; a tool that has low usability by physicians will always result in care that is less patient-centered. We suggest that holding companies accountable for assuring that their tool has a high degree of usability by physicians which will in turn facilitate greater patient-centric care.

\section{Acknowledgments}

Funding: None.

\section{Footnote}

Provenance and Peer Review: This article was commissioned by the Guest Editors (Naleef Fareed, Ann Scheck McAlearney, and Susan D. Moffatt-Bruce) for the series 
"Innovations and Practices that Influence Patient-Centered Health Care Delivery" published in Fournal of Hospital Management and Health Policy. The article has undergone external peer review.

Data Sharing Statement: Available at https://jhmhp. amegroups.com/article/view/10.21037/jhmhp-20-121/dss

Peer Review File: Available at https://jhmhp.amegroups.com/ article/view/10.21037/jhmhp-20-121/prf

Conflicts of Interest: All authors have completed the ICMJE uniform disclosure form (available at https://jhmhp. amegroups.com/article/view/10.21037/jhmhp-20-121/ coif). The series "Innovations and Practices that Influence Patient-Centered Health Care Delivery" was commissioned by the editorial office without any funding or sponsorship. The authors have no other conflicts of interest to declare.

Ethical Statement: The authors are accountable for all aspects of the work in ensuring that questions related to the accuracy or integrity of any part of the work are appropriately investigated and resolved. The study was conducted in accordance with the Declaration of Helsinki (as revised in 2013). The interview component of the telehealth study was the only portion considered research and was approved by institutional/regional/national ethics/ committee/ethics board of The Ohio State University and informed consent was taken from all participants.

Open Access Statement: This is an Open Access article distributed in accordance with the Creative Commons Attribution-NonCommercial-NoDerivs 4.0 International License (CC BY-NC-ND 4.0), which permits the noncommercial replication and distribution of the article with the strict proviso that no changes or edits are made and the original work is properly cited (including links to both the formal publication through the relevant DOI and the license). See: https://creativecommons.org/licenses/by-nc-nd/4.0/.

\section{References}

1. Kruse CS, Kristof C, Jones B, et al. Barriers to Electronic Health Record Adoption: a Systematic Literature Review. J Med Syst 2016;40:252.

2. Kruse CS, Mileski M, Alaytsev V, et al. Adoption factors associated with electronic health record among longterm care facilities: a systematic review. BMJ Open 2015;5:e06615.

3. Ajami S, Bagheri-Tadi T. Barriers for Adopting Electronic Health Records (EHRs) by Physicians. Acta Inform Med 2013;21:129-34.

4. Alami H, Lehoux P, Gagnon MP, et al. Rethinking the electronic health record through the quadruple aim: time to align its value with the health system. BMC Med Inform Decis Mak 2020;20:32.

5. Wong P, Panattoni L, Tai-Seale M. Features of PatientCentered Primary Care and the Use of Ambulatory Care. Popul Health Manag 2017;20:294-301.

6. Karsh BT, Beasley JW, Brown RL. Employed family physician satisfaction and commitment to their practice, work group, and health care organization. Health Serv Res 2010;45:457-75.

7. Street RL Jr. How clinician-patient communication contributes to health improvement: modeling pathways from talk to outcome. Patient Educ Couns 2013;92:286-91.

8. Street RL Jr, Liu L, Farber NJ, et al. Provider interaction with the electronic health record: the effects on patientcentered communication in medical encounters. Patient Educ Couns 2014;96:315-9.

9. Patel MR, Vichich J, Lang I, et al. Developing an evidence base of best practices for integrating computerized systems into the exam room: a systematic review. J Am Med Inform Assoc 2017;24:e207-15.

10. Asan O, Tyszka J, Crotty B. The electronic health record as a patient engagement tool: mirroring clinicians' screen to create a shared mental model. JAMIA Open 2018;1:42-8.

11. Asan O, Tyszka J, Fletcher KE. Capturing the patients' voices: Planning for patient-centered electronic health record use. Int J Med Inform 2016;95:1-7.

12. Fogarty CT, Winters P, Farah S. Improving patientcentered communication while using an electronic health record: Report from a curriculum evaluation. Int J Psychiatry Med 2016;51:379-89.

13. Kroth PJ, Morioka-Douglas N, Veres S, et al. The electronic elephant in the room: Physicians and the electronic health record. JAMIA Open 2018;1:49-56.

14. Arndt BG, Beasley JW, Watkinson MD, et al. Tethered to the EHR: Primary Care Physician Workload Assessment Using EHR Event Log Data and Time-Motion Observations. Ann Fam Med 2017;15:419-26.

15. Gardner RL, Cooper E, Haskell J, et al. Physician stress and burnout: the impact of health information technology. J Am Med Inform Assoc 2019;26:106-14.

16. Lander L, Baxter SL, Cochran GL, et al. Self-Perceptions 
of Readiness to Use Electronic Health Records Among Medical Students: Survey Study. JMIR Med Educ 2020;6:e17585.

17. Aaronson JW, Murphy-Cullen CL, Chop WM, et al. Electronic medical records: the family practice resident perspective. Fam Med 2001;33:128-32.

18. Graddy R, Reynolds SS, Wright SM. Coaching Residents in the Ambulatory Setting: Faculty Direct Observation and Resident Reflection. J Grad Med Educ 2018;10:449-54.

19. Rajaram A, Hickey Z, Patel N, et al. Training medical students and residents in the use of electronic health records: a systematic review of the literature. J Am Med Inform Assoc 2020;27:175-80.

20. Morrow JB, Dobbie AE, Jenkins C, et al. Firstyear medical students can demonstrate EHR-specific communication skills: a control-group study. Fam Med 2009; 41:28-33.

doi: 10.21037/jhmhp-20-121

Cite this article as: Sieck CJ, Henriksen B, Scott S, Kurien N, Rastetter M. Training to improve patient-centered electronic health record (EHR) use. J Hosp Manag Health Policy 2022;6:6.
21. Lee WW, Alkureishi ML, Wroblewski KE, et al. Incorporating the human touch: piloting a curriculum for patient-centered electronic health record use. Med Educ Online 2017;22:1396171.

22. Medicine So'ToF. Covid 19 resources. Society of Teachers of Family Medicine. 2020. Available online: https://www. stfm.org/teachingresources/covid19resources/. Accessed 08.20.2020.

23. Center TOSUWM. Center for Primary Care Innovation and Transformation. 2020. Available online: https:// wexnermedical.osu.edu/departments/family-medicine/pcit. Accessed 08.25.2020.

24. Adler KG, Edsall RL. EHR Switch Survey: Responses From 305 Family Physicians. Fam Pract Manag $2015 ; 22: 13-8$. 